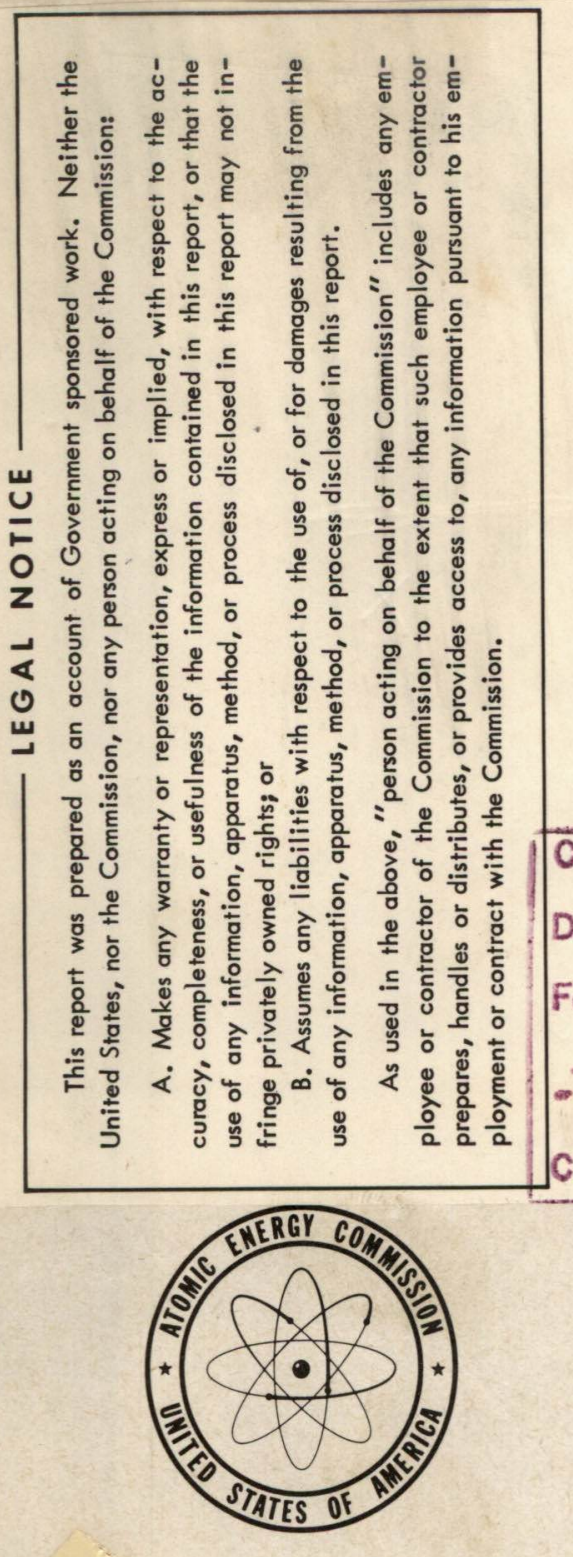

\section{RESTRLOED DATA}

This document contains restricted data as defined in the Atomic bresy Act of 1954 . Its transmittal or the desolssure of its contents in any manner to an unauthorized

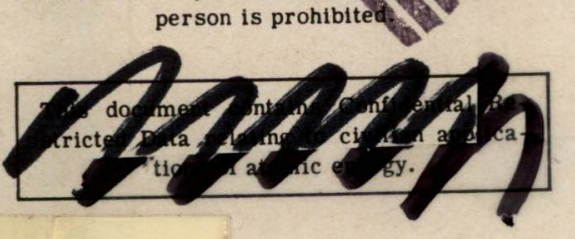

CLAS FIFICATION CANCELLED DATE $11 / 14 / 61$

For The Atomic Energy Commission

chief, $\frac{\text { Peclassification Branch a }}{\text { September 22, } 1955}$

\section{CONFIDETALL}

UNITED STATES ATOMIC ENERGY COMMISSION

STUDIES OF CORE FLOW BY ELECTROLYTE INJECTION AND BY DYE INJECTION

By

J. M. Trummel

E. C. Lindly

Oak Ridge National Laboratory

Oak Ridge, Tennessee SCASIFA FIRN GHANGSO TOT

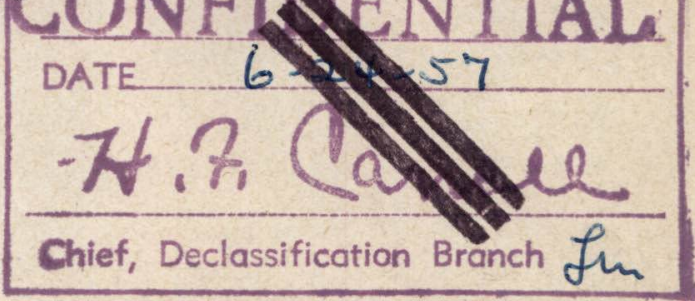

Technical Information Service Extension, Oak Ridge, Tenn.
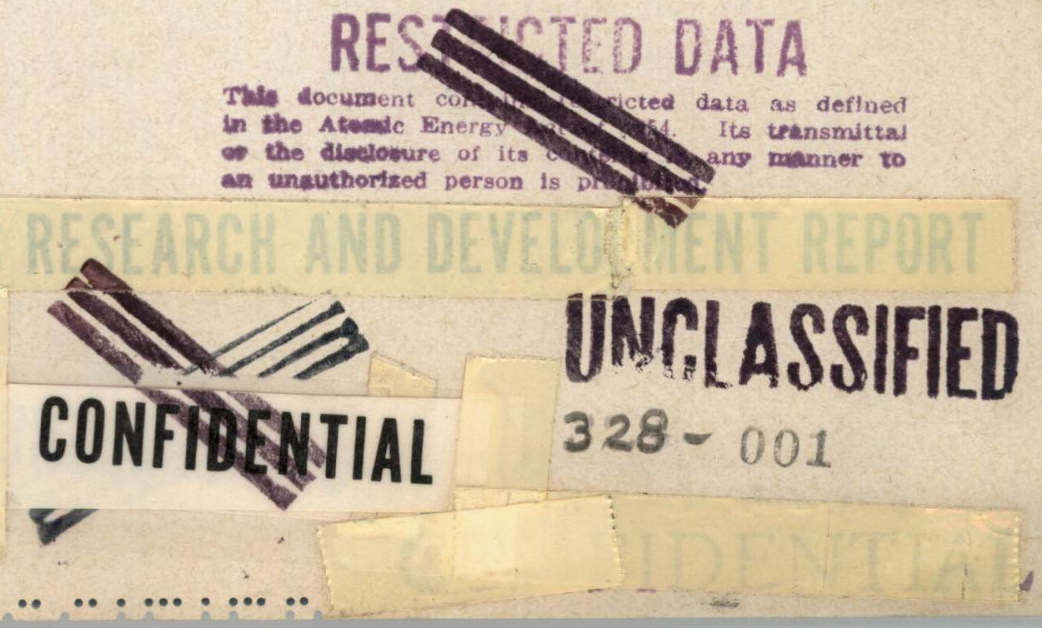

Photostat Charge \$4,59 for Access Permittees

Available from

Technical Information Service Extension P. O. Box 1001, Oak Ridge, Tennessee

\section{Technical Information Service Extension, Oak Ridge, Tenn.}




\section{DISCLAIMER}

This report was prepared as an account of work sponsored by an agency of the United States Government. Neither the United States Government nor any agency Thereof, nor any of their employees, makes any warranty, express or implied, or assumes any legal liability or responsibility for the accuracy, completeness, or usefulness of any information, apparatus, product, or process disclosed, or represents that its use would not infringe privately owned rights. Reference herein to any specific commercial product, process, or service by trade name, trademark, manufacturer, or otherwise does not necessarily constitute or imply its endorsement, recommendation, or favoring by the United States Government or any agency thereof. The views and opinions of authors expressed herein do not necessarily state or reflect those of the United States Government or any agency thereof. 


\section{DISCLAIMER}

Portions of this document may be illegible in electronic image products. Images are produced from the best available original document. 

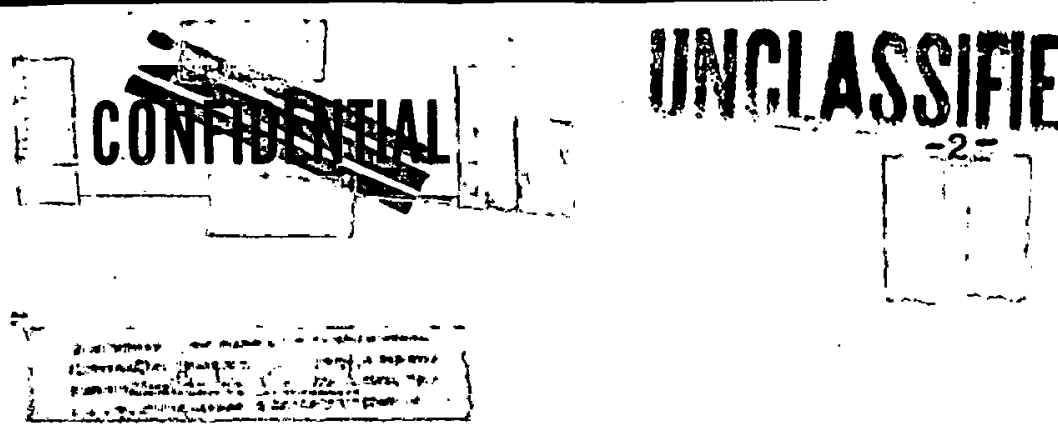

FOREWARD

Described herein are two test techniques used to obtain information on the Plow in two Core models. One of these techniques involves the measurement of electrical conductivity of water following electrolyte injection; the other involves taking motion pictures following injection of a coloring agent. Considerable information was obtained; however, no statement is warranted at this time in regard to the temperature field characteristic of the flow when a volume heat source is imposed. It is felt that these techniques can be developed so that some quantitative data on diffusivity can be obtained. Work in this direction is continuing.

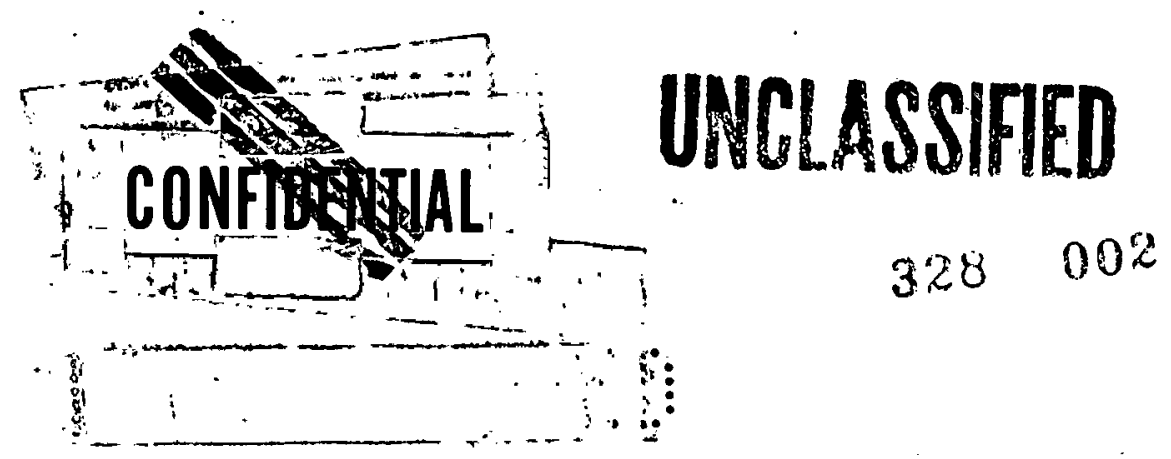


It seems reasonable that the eddy transfer of heat and electrolyte would be very similar to that of momentum transfer. Molecular diffusion refers to the transfer of such quantities as heat, matter and momentum without convection in the fluid. An analogous process is heat transfer by conduction or the spread of an electrolyte in a stagnant fluid.

In making the analogy between the diffusion of heat and the diffusion of electrolyte in turbulent flow it is reasonable to assume that diffusion by eddy transfer is completely similar; however, the molecular diffusion rates are not similar. Thermal diffusivity of the fluid of interest is $7.7 \times 10^{-6} \mathrm{ft}^{2} / \mathrm{sec}$. while the molecular diffusivity of the electrolyte in water is about $1.2 \times 10^{-8} \mathrm{ft}^{2} / \mathrm{sec}$.

It is evident that the electrolyte injection method yields information on only the eddy diffusion of heat. Since thermal diffusivity is the controlling mechanism where eddy diffusion is small or zero as in laminar flow near the containing surfaces, no valid information on heat transfer near the wall can be obtained by electrolyte injection. However, some information can be obtained on the flow feeding into the boundary layer due to entrance conditions and the sweeping of the boundaries by eddies. This assumes that electrical conductivity devices can be brought near the wall without significantly disturbing the flow.

The diffusion of a color changing agent in the water flow is presumed to be similar to the diffusion of electrolyte. Practically there are differences in observation since it is difficult to differentiate between color densities directly by eye or by looking at moving picture of the flow. Further, the moving pictures give an over-all pattern of flow. Since the color change used involved a chemical process with some 

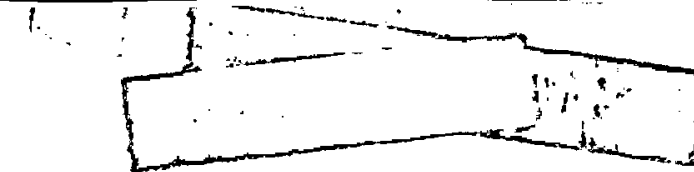

threshold concentration, one observed the advance of a front of rather low concentration and from this some notion of diffusion rates was obtained. However, as the front progressed it diffused and the concentration drops below that required for color change and the front is lost. Thus fluid transit times from analysis of moving pictures permits determination of velocities at various points of injection.

Flow similarity between the water flow and flow of fluid in question was obtained by using equal Reynolds numbers. This requires a correction in processes dependent of velocity $2 \mathrm{~s}$ is discussed later.

\section{APPARATUS}

Apparatus was devised for making conductivity measurements in the flow in two existing facilities. One of these facilities was a full-scale model constructed of aluminum; the other was a full-scale model constructed of transparent plastic. Sketches of these two models are shown in Figure 1 and Figure 2. Access holes for $3 / 16$ inch diameter probes were available at two meridians and various levels as shown.

Water is circulated through the aluminum model by a single pump and the discharge is divided to supply two header inlet lines. Valves and orifice meters in each line pormit balainlng the llow into the header. Water discharges from the model into a 3000 gallon tank and is recirculated by the pump:

Water is circulated through the transparent model by two pumps arranged as shown in Figure 2. Water is recirculated at a rapid rate;

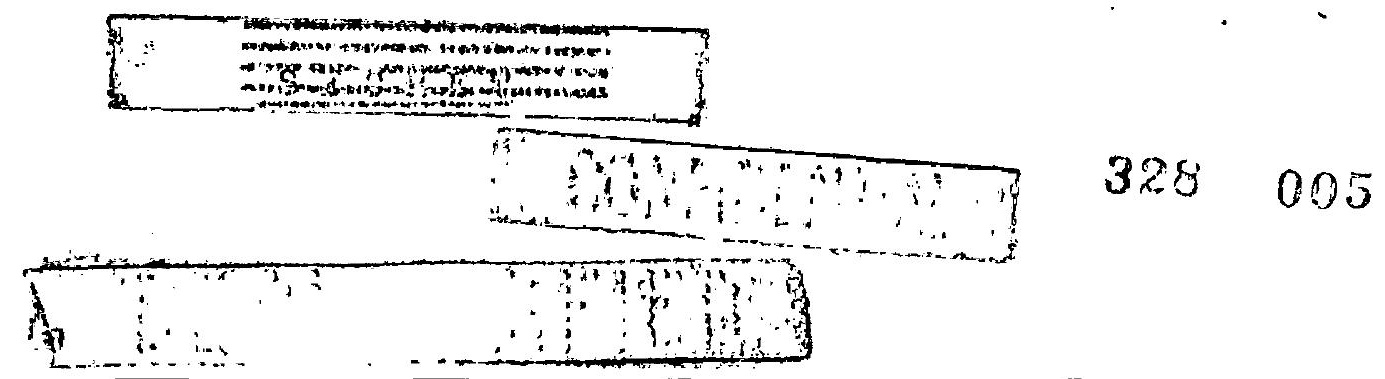



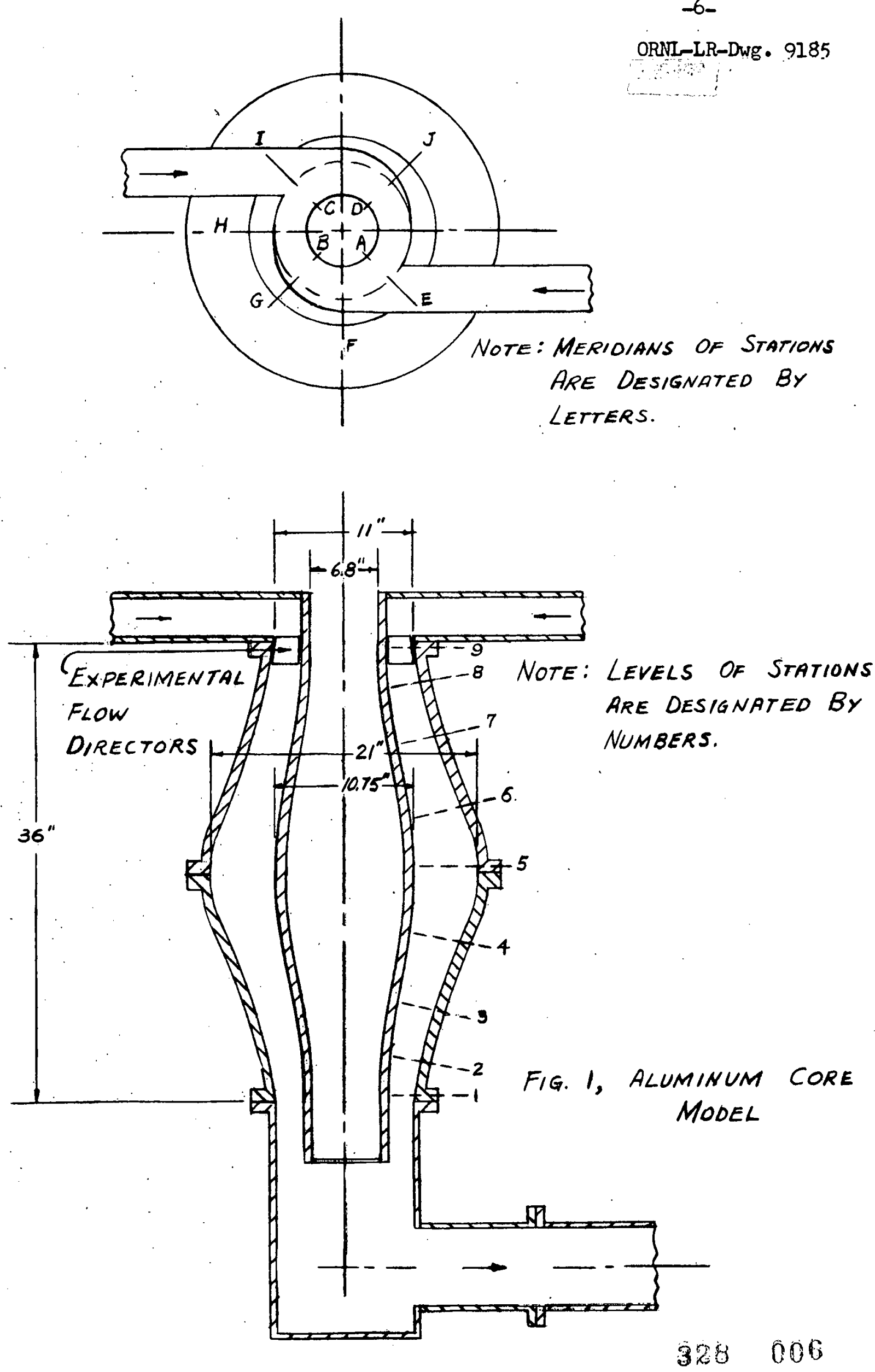

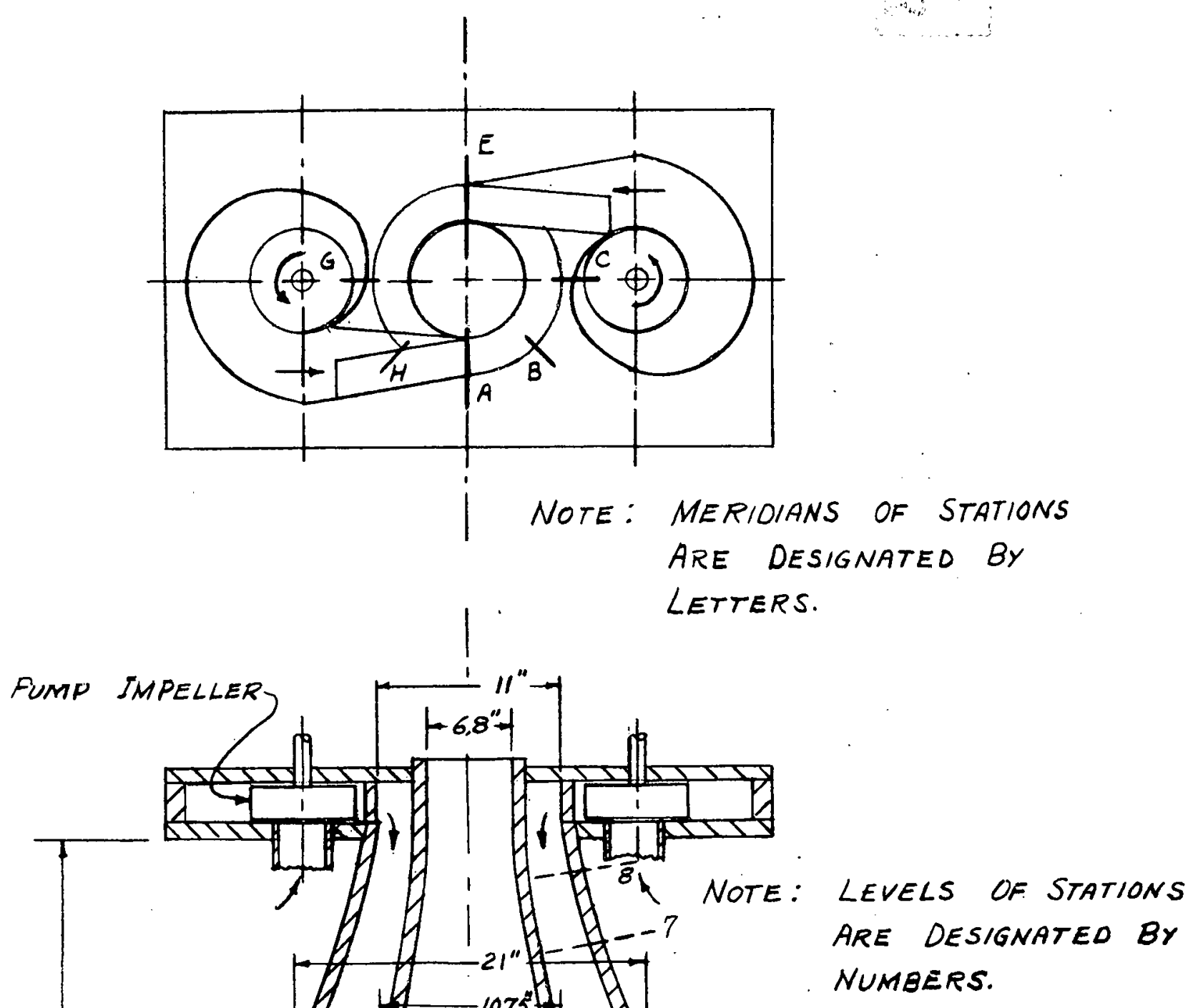
the active volume of system is $7.5 \mathrm{ft}^{3}$ and the pumping rate $1.8 \mathrm{ft}^{3} / \mathrm{sec}$.

A schematic sketch of the conductivity measuring and recording apparatus is shown in Figure 3, The basic sensing circuit is a wheatstone bridge with two fixed legs of 10,000 ohms, a variable leg and the leg which is the conductivity probe. With this arrangement it is possible to balance the bridge for initial conductivity of the water; then any change in conductivity of water between probe electrodes causes a bridge unbalance and a consequent electrical signal to the amplifier. The sensitivity of the bridge expressed in volts per ohm change in probe resistance is a function of initial probe resistance as shown in Figure 4. A sample of water used in the tests had a resistance of 2500 ohms. From this it can be seen that the sensitivity is $29 \times 10^{-4}$ volts/ohm. From calibration data the signal from a concentration change of 20 milligrams of sodium chromate in $1000 \mathrm{cc}$ of water used was 0.59 volts. The corresponding change in probe resistance is about $205 \mathrm{ohms}$, so one milligram of sodium chromate in $1000 \mathrm{cc}$ of water is worth about 10 ohms under conditions of the test. It should be noted that this relation is a good approximation for this test, but that in general the relation between concentration and resistance is not linear. Precise determination of the resistance corresponding to various electrolyte concentrations was not possible due to polarization effects on the probe electrodes.

The use of alternating current of $1000 \mathrm{cycles} / \mathrm{second}$ or higher should eliminate polarization effects; however, additional instrumentation would be required and some problems of balancing capacitances likely would be encountered.

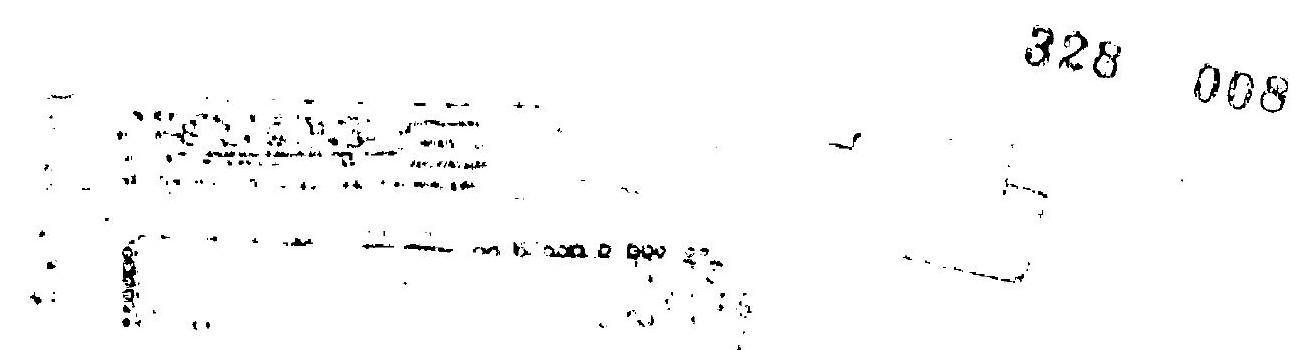


ORNI-LR-Dwg • 9187 UNCLASSIFIED

10,000 OHM HELIPOT
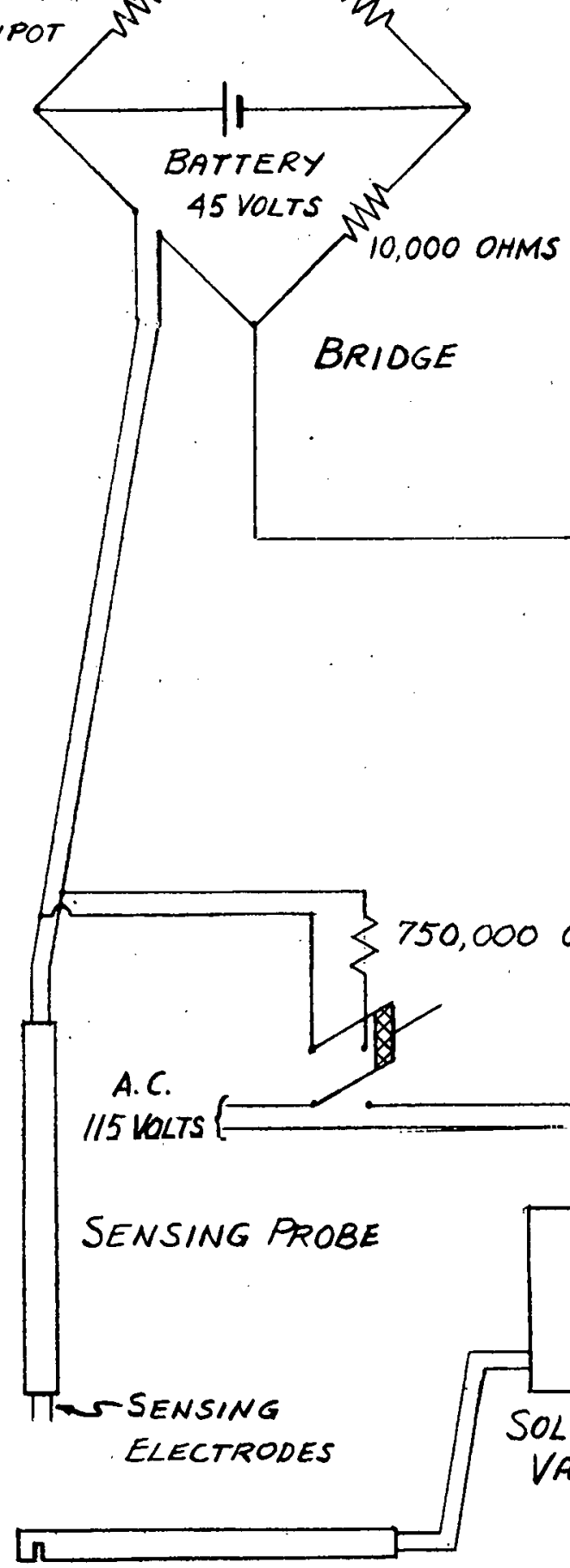
PROBE

TO SECONO PROBE CIRCUIT.

10,000 OHMS 
$-10-$

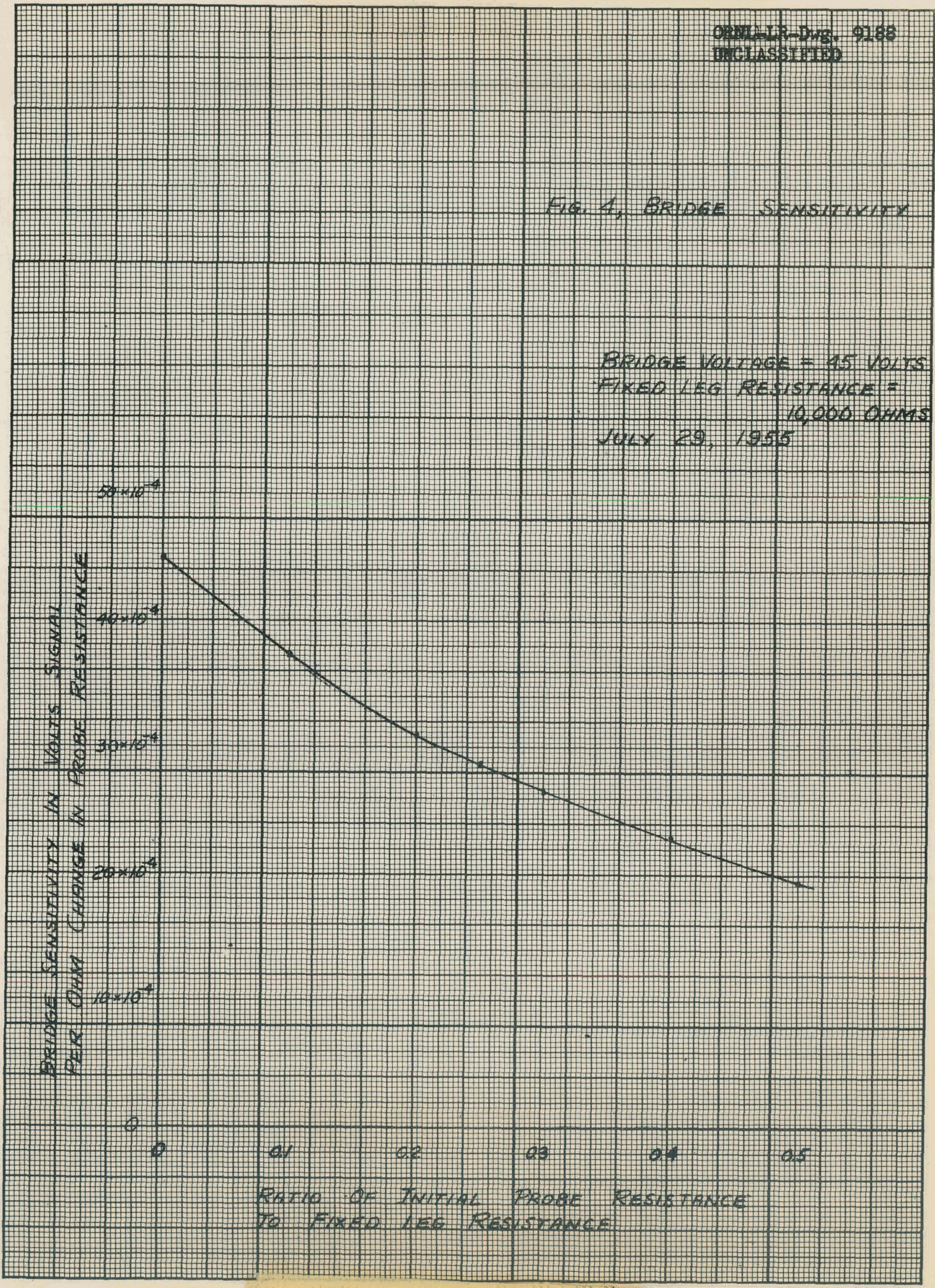


Considerable effort was required to devise a satisfactory conductivity probe. The final construction used is shown in Figure 5. Some corrosion of the Kovar electrode wires occurs but life is considered satisfactory. Very rapid corrosion at the wire-to-glass seal was eliminated by coating the seal and about $1 / 8$ inch of the wire with radio cement. Injection of electrolyte into the flow was first made manually with a glass syringe. Usual injection was $5 \mathrm{cc}$ of nearly saturated sodium chromate solution over a period of about $1 / 2$ second. Later a pneumatic injector was devised as shown schematically in Figure 3. This permitted injection as short as $1 / 5$ second. In addition, a circuit was devised to place an injection signal on the Brush record.

Little additional apparatus was required for the moving pictures taken of flow in transparent model. The Y-12 photographer used a $16 \mathrm{~mm}$ camera running at the rate of 64 frames per second. Injection of color changing agent was made manually with a glass syringe. In order to obtain an intense color change with minimum injection of material, the starch- . iodine reaction was used. Approximately 80 grams of soluble starch dissolved in warm water was injected into the water. To obtain a dense black color about $10 \mathrm{cc}$ of methyl alcohol saturated with elemental iodine was injected. The color was neutralized by injecting a solution of sodium thiosulfate. This sequence of injections could be repeated many times.
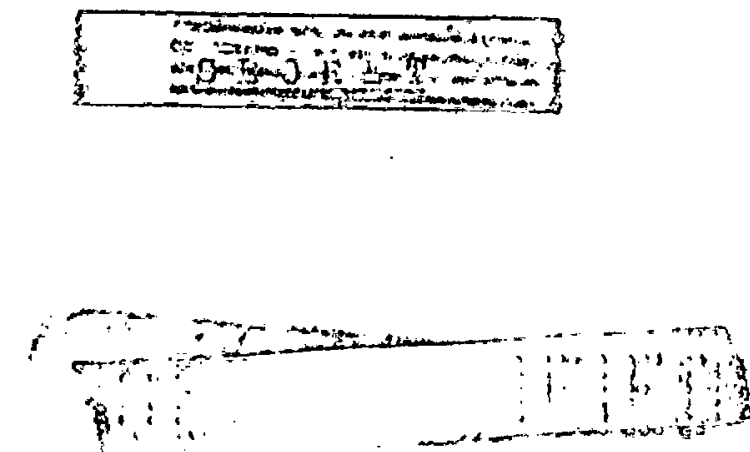
ORNL-IR-DWE . 9189

UNCLASSIFIED

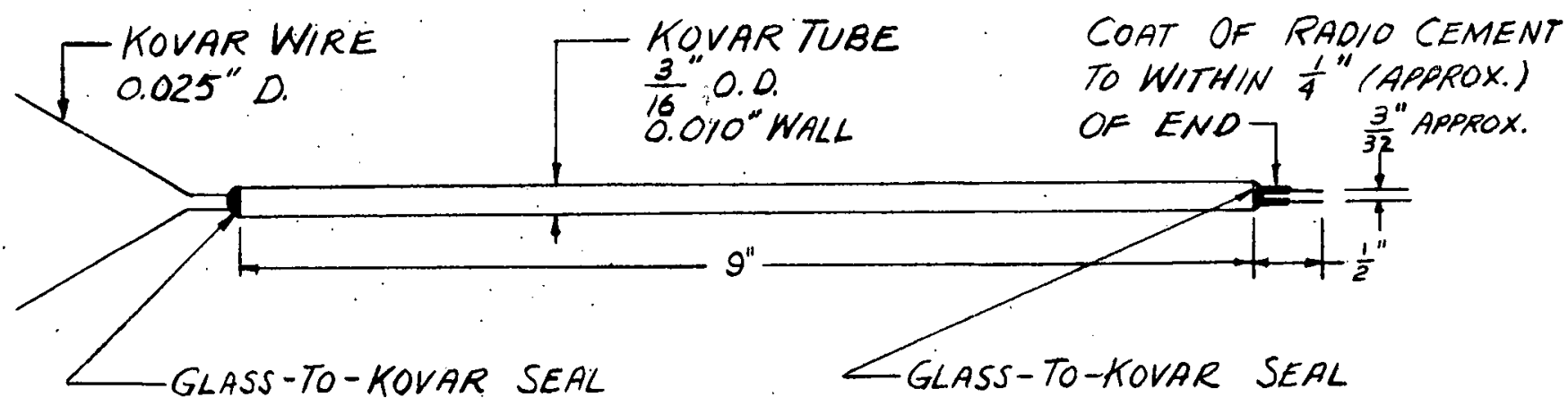

ELECTRICAL CONOUCTIVITY PROBE

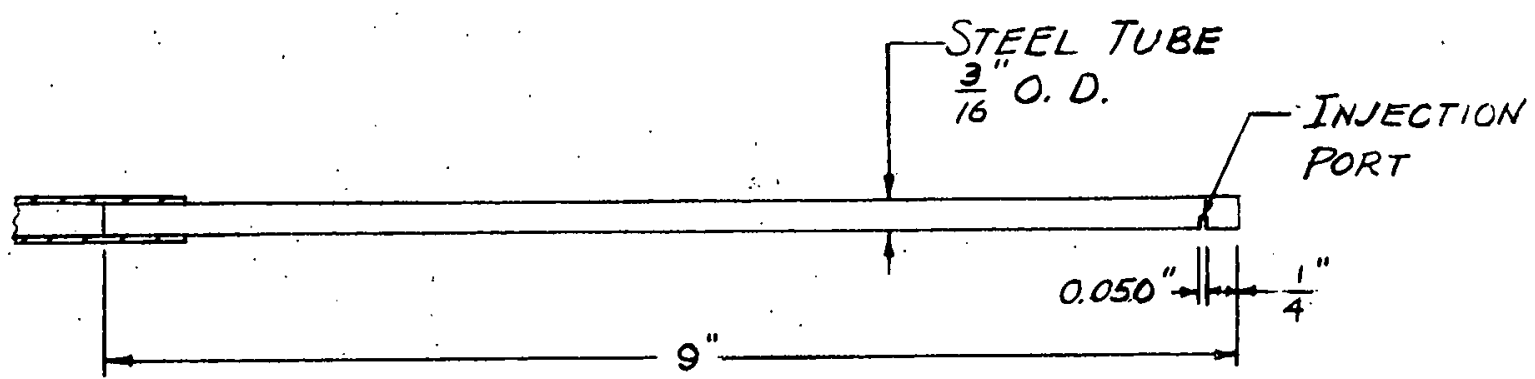

INJECTION PROBE

Fig. 5, electrical CONDUCTIVITY PRobe and INJECTION PROBE 
TESTS

Electrolyte injection experiments were conducted on the aluminum model with turning vane series No. 8 and No. 9 and on the plastic model with an inlet header designed to produce a meshing system of vortices. Each test requires the solution of two conductivity measurement points and a point of injection. In addition, the injection may be short ( $1 / 5$ to $1 / 2$ second) or sustained so that equilibrium concentrations are obtained.

A large number of combinations of the two probe locations and electrolyte injection locations was available. In most cases information from velocity probe tests was used to determine conductivity probe locations. Also tests were run with one probe at inlet and one probe at outlet in order to determine the distribution of transit times for the flow. A total of 111 recorded injections were made. Each test was numbered for identification and information on probe locations, point of injection, chart speed, and kind of injection was recorded on a $\mathrm{log}$ sheet.

Further details of each test are best presented later with the discussion of results.

Approximately 100 feet of $16 \mathrm{~mm}$ movie film was exposed at the rate of 64 frames per second in making moving pictures of the flow in the transparent model. A series of nine tests were photographed. Two color changes were produced; one by the injection of iodine into the clear 
starch solution and one by the injection of sodium thiosulfate into darkened iodine-starch solution. Subsequent viewing of the film indicated the darkening process to be more informative.

Each moving picture sequence was identified by a number appearing on the film. These tests were as follows:

Run No. 1. Injection of iodine into suction leg of left-hand pump.

Run No. 2. Injection of sodium thiosulfate into suction leg of lefthand pump.

Run No. 3. Injection of iodine at station 7H next to island.

Run No. 4. Injection of sodium thiosulfate at station 7H next to island.

Run No. 5 and 5 A. Injection of iodine at station $7 \mathrm{H}$ near outer wall.

Run No. 6. Injection of sodium thiosulfate at station $7 \mathrm{H}$ near outer wall.

Run No. 7. Injection of iodine at station $7 \mathrm{H}$ in middle of channel.

Run No. 8. Injection of sodium thiosulfate at station $7 \mathrm{H}$ in middle of channel.

Run No. 9. Short injections of iodine at station 7H in middle of channel.

The station locations may be determined from Figure 2.

Typical conductivity records obtained following electrolyte

injection are shown in Figure 6. One of these is for a short injection and the other for a sustained injection.

One method used to analyze the conductivity record is that of plotting the fraction of total area under the conductivity curve against 
time measured from time zero at first indication of electrolyte at the inlet to the core. Such curves are shown in Figure 7 and Figure 8. Essentially the same information is presented in Figure 9 and Figure 10 in which the derivatives of the curves of Figure 7 and Figure 8 are plotted. These derivative functions are similar to probability density functions and are amenable to description by mathematical functions. Important information readily obtained from these graphs are the maximum and minimum transit times between probe stations as well as the mean transit time. A check can be made on this mean transit time from flow rate and core volume.

The evaluation of these distributions in terms of some measure of diffusion has not yet been achieved. If one assumes uniform diffusivity and uniform velocity across each section then it should be possible to ascertain the theoretical form of the distribution. Work on this is continuing. It appears that these distributions would be closely related to the "normal" distribution. 1,2

1. Jacob, Max, Heat Transfer . John Wiley \& Sons, 1950, Vol. 1, p.253.

2. Kalhahe, A. A., Fluid Mechanics and Statistical Methods in Engineering, Investigation of Liquid Turbulence and Suspended Material Transportation. University of Pennsylvania Press. 

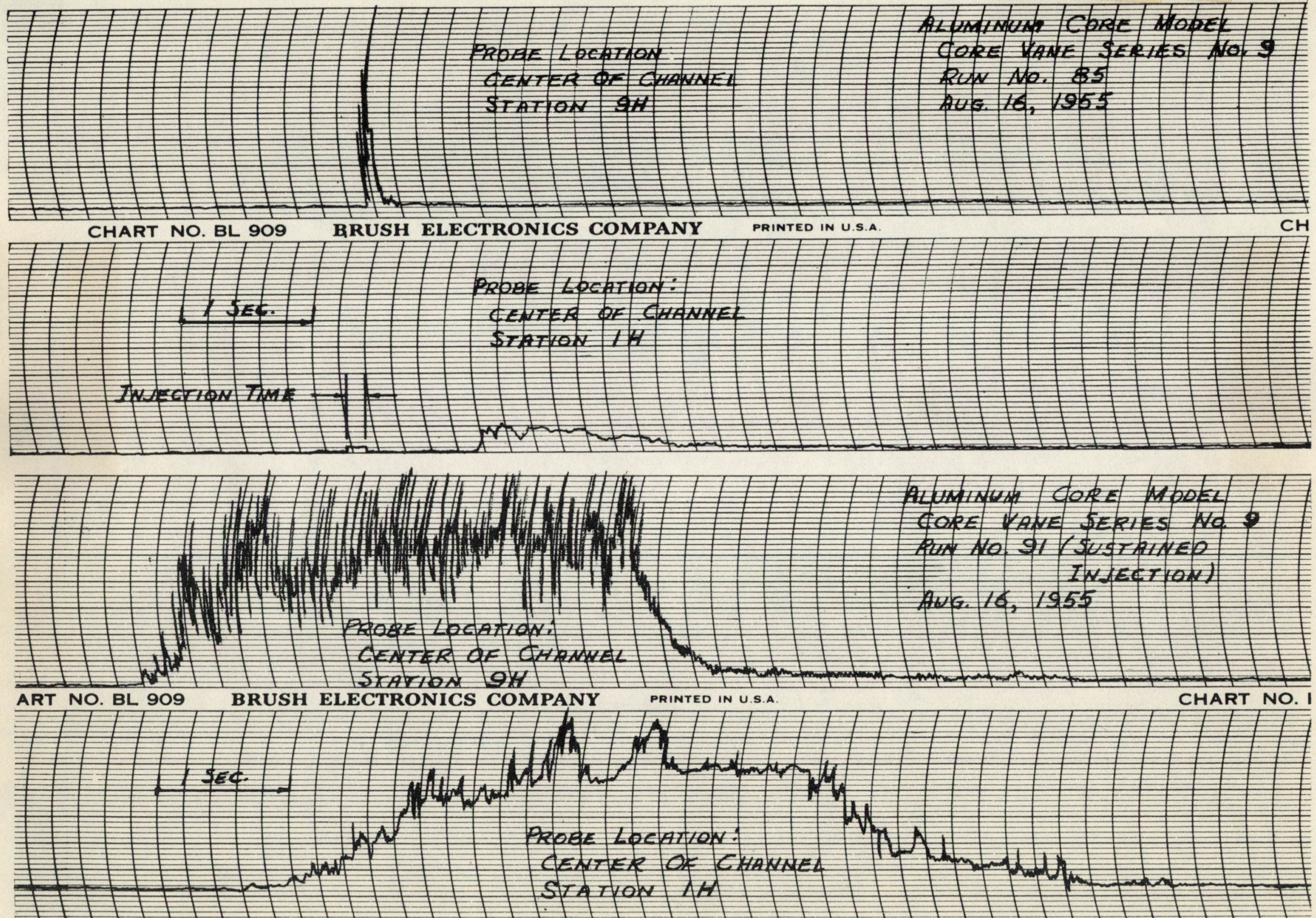
ORNL-LR-Dwg • 9191

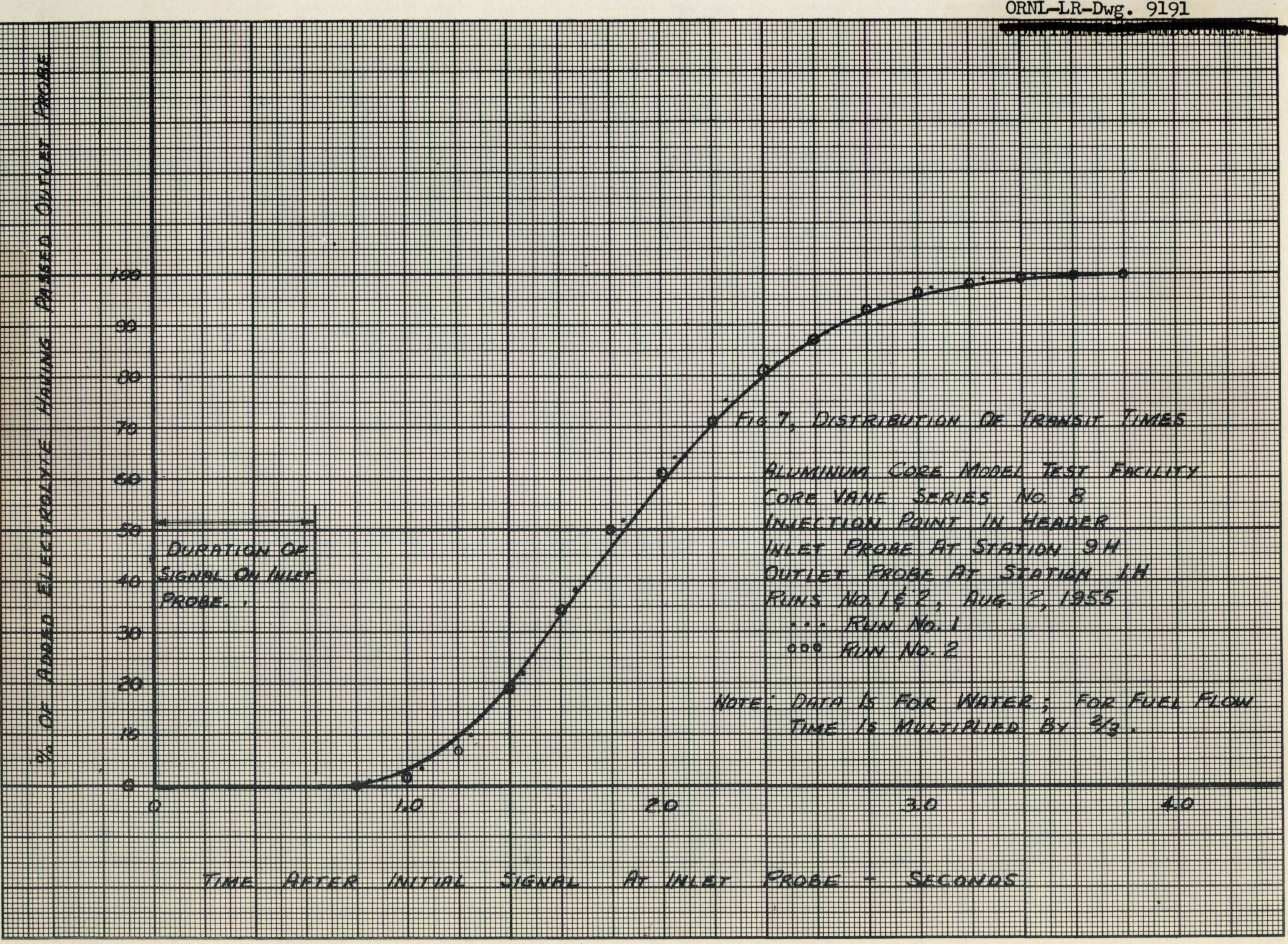


ORNL-LR-Dwg. 9192

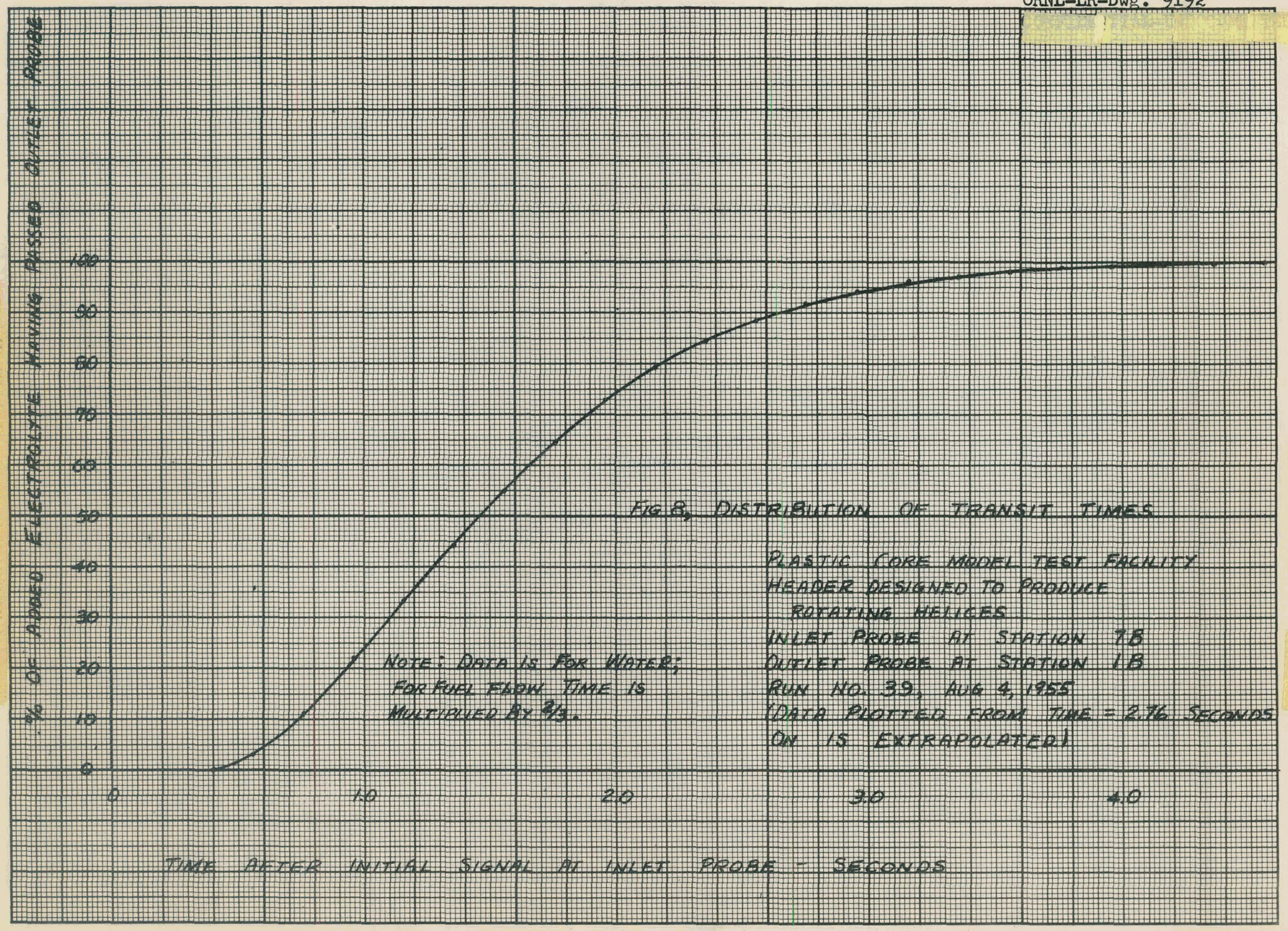


ORNL-LR-Dwg • 9193

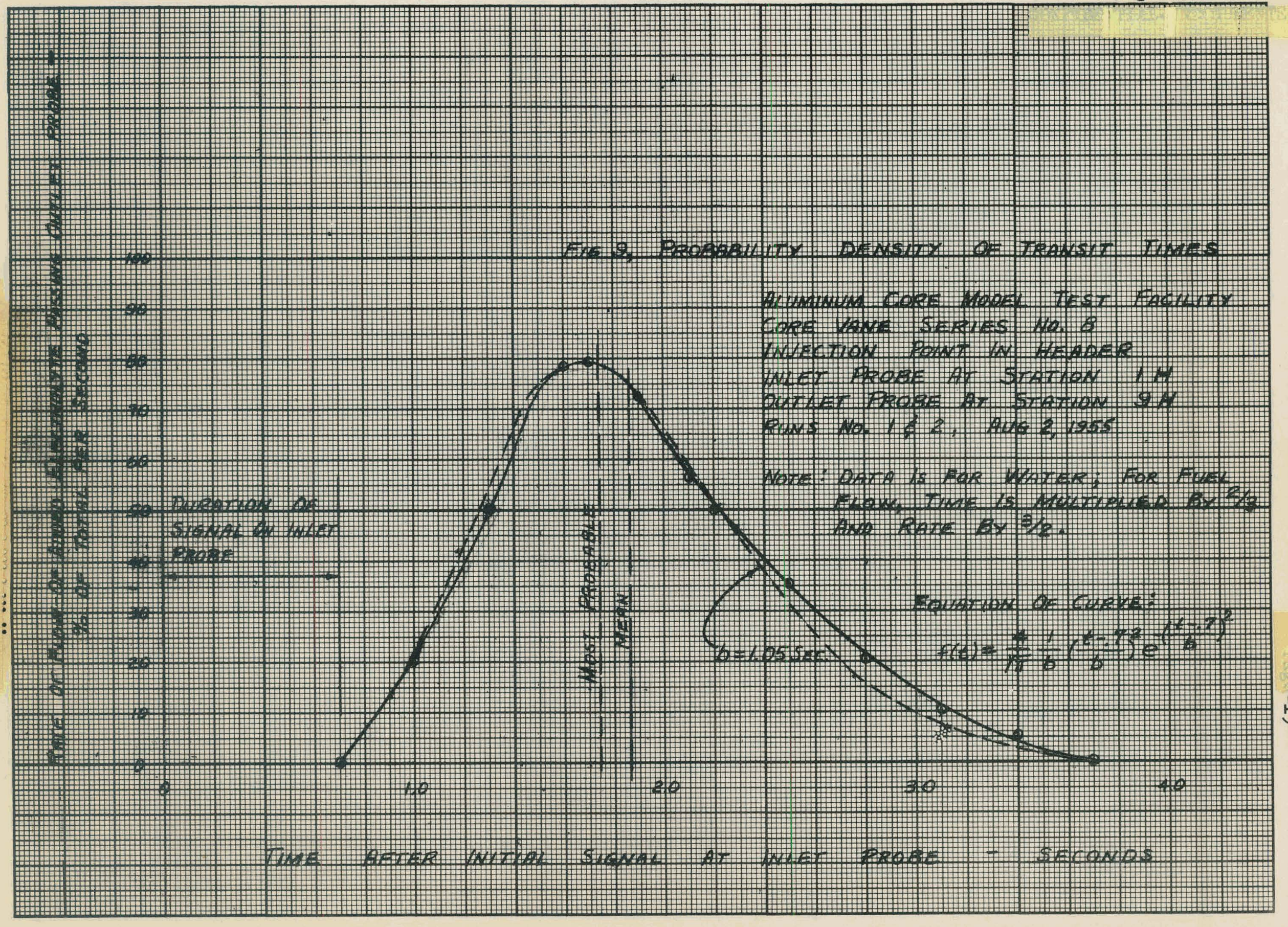


ORNL-LR-Dwg • 9194

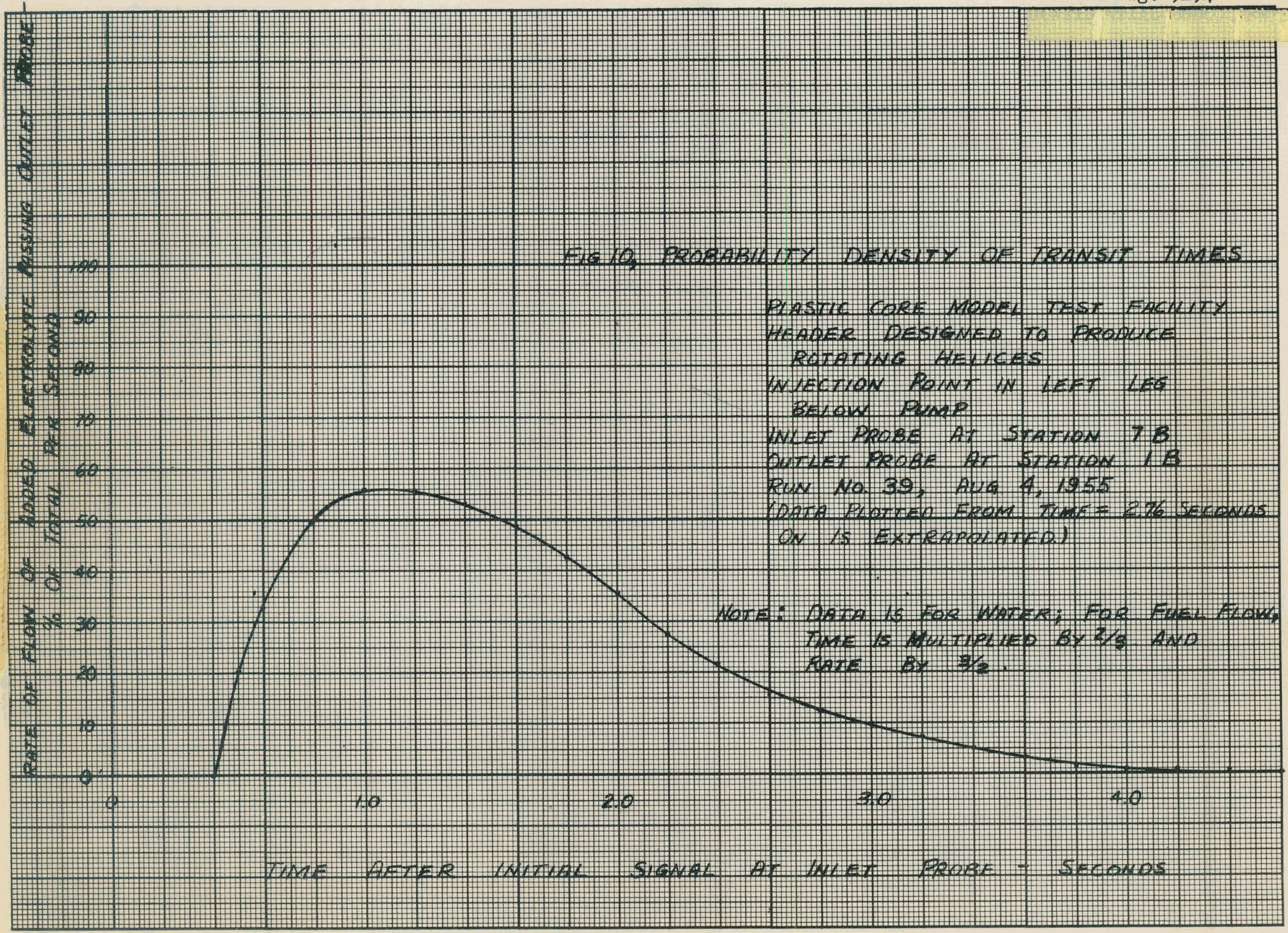


The "spread" of this distribution is some function of diffusivity in the main direction of flow. Such spread is undoubtedly also due to non-uniform velocity in the actual case. Moreover, much of this diffusion is normal to the mean local velocities since many of these velocities have large peripheral components. So far it has not been possible to separate these various effects though it should be possible to separate some of them.

In these water flow tests the flow rates were $2 / 3$ of those proposed for fuel flow in order to obtain the Reynolds number of the full flow. This satisfies the requirement of similarity of flow pattern and consequentIy the ratio of eddy size (length) to model characteristic length should be the same for water flow as for fuel flow. Further, the ratio of some measure of eddy velocity to mean stream velocity should be the same for the water flow as for fuel flow. Finally then, the diffusivity (kinematic eddy viscosity) and the mean velocity should be proportional for a fixed Reynolds number. Thus the kinematic eddy velocity obtained from water flow should be multiplied by $3 / 2$ to apply to fuel flow with the same Reynolds number. One way to make this adjustment is to change the time scale on the curves of Figures $7,8,9$ and 10 as noted.

Conductivity records following sustained infection of electrolyte were examined for evidence of varying rates of diffusivity between the 
main stream and other regions of the flow. Stated another way this examination was to determine the presence of relatively isolated recirculatory volumes in the flow. Some evidence of two different rates of decay is seen in run No. 91 of Figure 6.

Other information on the paths of a particle flowing through the core was obtained by injecting electrolyte at selected stations and looking for electrolyte at other stations. Since the number of probes is limited. to two this is a slow process. Evidence of reverse flow can be obtained in this way though such evidence can also be obtained by moving picture photography and by velocity probe technique.

Analys is of the moving pictures was made by projection at a nominal rate of 16 frames per second. This extended the time scale for water flow by a factor of 4 and the time scale for fuel flow by a factor of 6 .

Frame-by-frame analysis also was made by projection with a time and motion- study projector. By this method minimum and maximum transit times were observed, some local velocities determined and rate of spread of dye observed.

\section{RESULTS}

Transit Times. Two distributions of transit times for the aluminum model, turning vane series No. 8, are plotted in Figure 7 and Figure 9. Similar distributions for the lucite model with header designed to producing meshing vortices are shown in Figure 8 and Figure 10. Minimum and 
maximum transit times are summarized below:

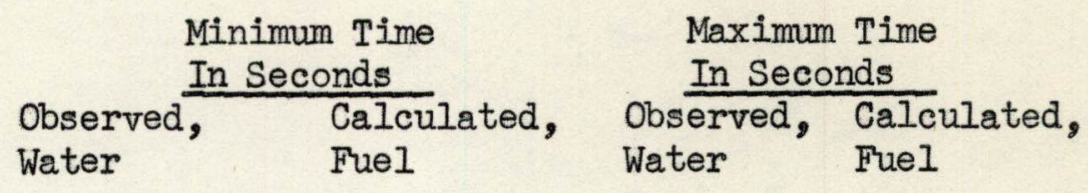

Aluminum model

Vane series No.8

0.70

.47

3.0

2.0

Aluminum model

Vane series No. 9

0.77

0.51

3.2

2.1

Lucite model*

By Electrolyte

0.40

0.27

3.8

2.5

Lucite model

By Photography

0.50

0.33

2.0

1.3

*These times from level 8 to outlet while times for aluminum core are from level 9 to outlet.

The difference between photographic times and electrolyte times is due to neutralization of the color front and rear. This neutralization has been observed directly.

The observed times as recorded for vane series No. 9 are averages of 8 runs. Root mean square of deviations from mean for maximum transit time is 0.36 seconds.

Assuming a normal distribution will describe the observation differences then the probable error of the mean values is .012 seconds for the minimum transit time and .09 seconds for the maximum transit time.

Decay Curves. A series of sustained electrolyte injections were made for probe locations in aluminum model at meridian $\mathrm{H}$ for vane series No. 9. For each location a record was made for the probe end $1 / 8$ inch 
from island and for the probe and $1 / 4$ inch from the outer wall. The times required for the concentration to decay from equilibrium injection value to $1 / 2$ of this value and then to decay to zero were determined. The curves were smoothed to obtain these data. The times are tabulated below:

\section{DECAY TTME}

\begin{tabular}{llll} 
Run No. & Probe Position & To I/2 Value & To Zero \\
Seconds & Seconds \\
\hline 98 & $7 \mathrm{H}$, near Island & 0.60 & 1.8 \\
99 & $7 \mathrm{H}$, near Outer wall & 1.0 & 2.6 \\
100 & $6 \mathrm{H}$, near Island & 0.75 & 1.95 \\
101 & $6 \mathrm{H}$, near Outer wall & 0.70 & 2.2 \\
102 & $5 \mathrm{H}$, near Island & 0.70 & 1.6 \\
103 & $5 \mathrm{H}$, near Outer wall & 0.80 & 1.6 \\
104 & $4 \mathrm{H}$, near Island & 0.80 & 3.2 \\
105 & $4 \mathrm{H}$, near Outer wall & 0.92 & 3.2 \\
106 & $3 \mathrm{H}$, near Island & 1.9 & 4.0 \\
107 & $3 \mathrm{H}$, near Outer wall & 1.6 & 4.0 \\
108 & $2 \mathrm{H}$, near Island & 1.6 & 3.1 \\
109 & $2 \mathrm{H}$, near Outer wall & 1.2 & 3.4 \\
96 & $1 \mathrm{H}$, near Island & 1.5 & 3.8 \\
97 & $1 \mathrm{H}$, near Outer wall & 1.6 & 3.6
\end{tabular}




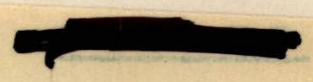

In general these decay times should increase as probes are moved from level 7 down through station 1. A decay time greater than any below it would indicate a relatively poor local diffusion from the upper location. There is a marked increase in these times between stations 5 and 4 and stations 3 and 2. This suggests that the diffusion from stations 4 and 3 is relatively poorer than from the remainder of the flow volume sampled, and is slightly worse at the outer wall. Another relatively poor station is at $7 \mathrm{H}$ near the outside wall. Diffusion is greater at $7 \mathrm{H}$ near the island than at outside wall. It is known from velocity probe data that the island surface is swept by an up-flow of considerable velocity.

Although the information cited above is for the aluminum model with vane series No. 9, some of the characteristics of flow below level 5 seems consistent with observations made on the plastic model. Study of moving pictures indicates a high degree of mixing in the upper half of the core. Just below the equator there seems to be a change to a flow regime in which rotational effects predominate (velocity vectors at the equator are nearly horizontal.) As the fluid is accelerated into the converging section it appears that high velocity streamers of color leave the main bulk and move along the island wall to outlet. The color dwells between station 4 and outlet for a noticeably longer time than in the upper hemisphere. On the outside wall the region of lowest diffusivity appears to be in the region of stations 3 and 4. 
Some Results From Moving Pictures. Much of the results of moving picture analysis has been cited previously in comparison with electrolyte injection data. An important advantage of the picture technique is that an over-all picture of the flow is presented so that other methods of investigation may be applied to areas of interest. Some quantitative data are obtained from the film; the transit times have been given earlier. Two velocities were determined. A "chunk" of dye was observed just below level 5 with a velocity of $16 \mathrm{ft} / \mathrm{sec}$. horizontal. The streamers leaving the flow below the equator were about $1-1 / 4$ inches in diameter with an angle about 30 degrees from horizontal. The leading edge of these streamers is lost by diffusion and/or chemical neutralization. Dye is carried around one complete revolution from injection at station 7 in 18 frames or about $1 / 3$ second. After this revolution the leading edge is diffused so as to cover the northern hemisphere. This figure of 18 frames was quite consistent for injections made at $7 \mathrm{H}$ whether near island, near outer wall or at midchannel. This time is also consistent with conductivity records which indicate times slightly greater than 0.3 second.

Conclusions and Recommendations. As stated earlier the ultimate purpose of these experiments was to obtain information which would assist in predicting the temperature field resulting when volume source heating is imposed. As yet no quantitative data can be presented for this purpose. 


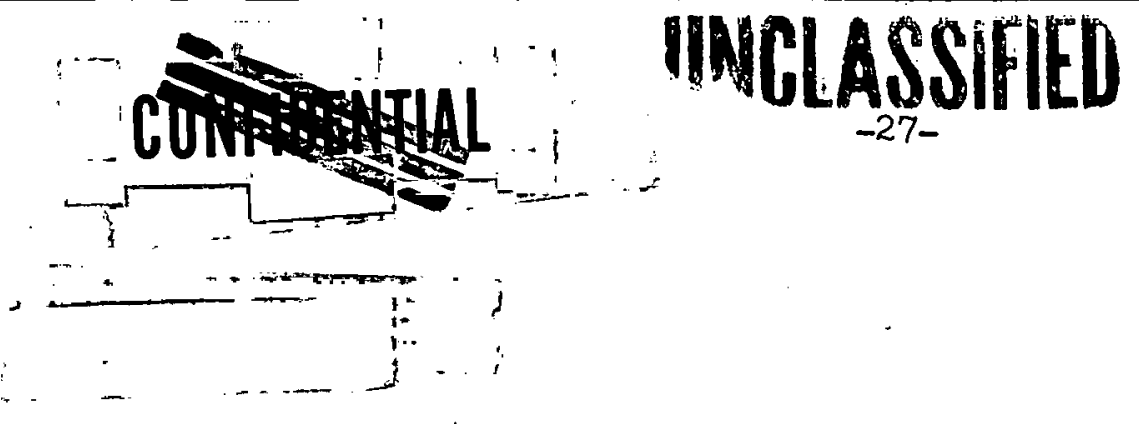

It is the writer's opinion, based on data discussed earlier, that the region of poorest diffusional characteristics for the geometries tested is just below level 5 (equator) and that this is due to the characteristic of a mainly centrifugal flow entering a converging section. However, it may be that the diffusion with this centrifugal component is greater than it would be with pure axial flow due to higher local velocities resulting when the centrifugal component is superimposed on the axial or through-flow component.

It is felt that both techniques can be further refined both in instrumentation and analysis so that some quantitative data can be obtained. Work is continuing in this direction.
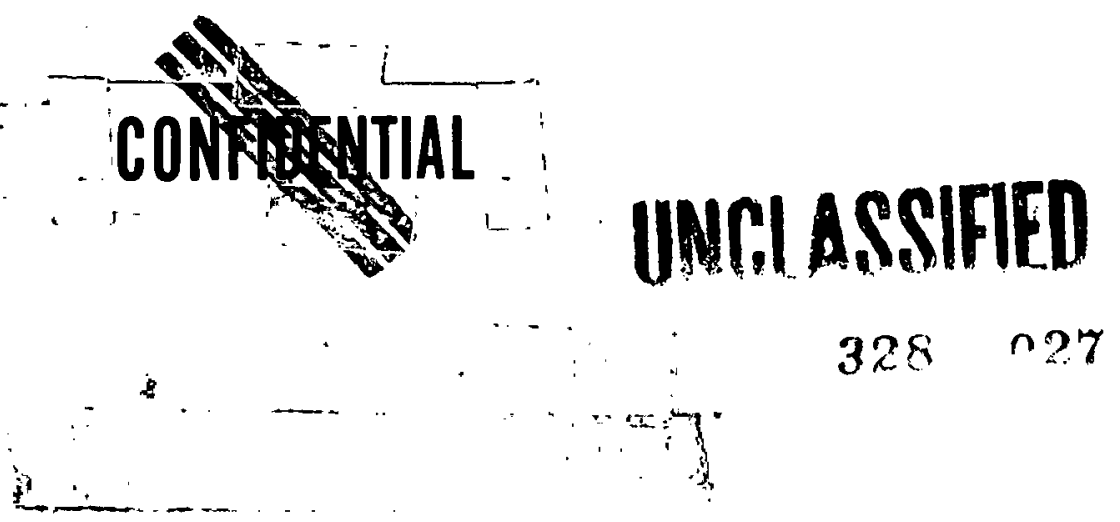